\title{
La formación del especialista en medicina crítica
}

\section{Training of the specialist in critical medicine}

\author{
Jorge A. Castañón-González, * Luis A. Gorordo-Delsol, Jessica Garduño-López, \\ Marcos A. Amezcua-Gutiérrez y María de los Á. Espino-Ángeles \\ Secretaría de Salud, Hospital Juárez de México, Unidad de Cuidados Intensivos de Adultos, Ciudad de México, México
}

La vertiginosa expansión de los conocimientos en todos los ámbitos (tecnología, organización, educación, investigación, administración, financiamiento de los servicios de salud, etcétera) afecta la formación y capacitación de los profesionales de la salud, ${ }^{1}$ lo que ha dado como resultado que las especialidades y subespecialidades médicas ya no solo se clasifiquen de acuerdo con la forma tradicional por aparatos y sistemas (neurología, nefrología, etcétera), sino también por la atención que dan a grupos etarios (pediatría, geriatría), género (ginecología y obstetricia), tipo de padecimientos (infectología, oncología), sitio de trabajo (anestesiología, medicina de urgencias), tipo de procedimiento (cirugía de colon y recto, neurofisiología, cirugía neurológica), tipo de atención (primaria o secundaria), por su estructura horizontal (medicina crítica, medicina familiar) o vertical, como las especialidades troncales

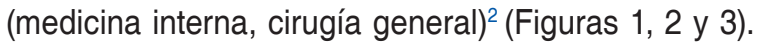

En la actualidad, la atención médica hospitalaria es de colaboración multidisciplinaria, en la cual la intervención del especialista o subespecialista es puntual, selectiva y generalmente por periodos cortos e intermitentes; se requiere la participación de otros profesionales de la salud que tienen una formación diferente, pero complementaria. Para lidiar con la escases de especialistas (de la cual no se vislumbra una solución a corto plazo), algunas especialidades, en particular medicina crítica, la subsanan de dos formas:

- Adquisición de destrezas técnicas que eran exclusivas de otras especialidades, como la ecografía para guiar procedimientos, realizar evaluación neurológica, hemodinámica y respiratoria; broncoscopias, terapia de reemplazo renal, etcétera.
- Generación de programas de entrenamiento de posgrado mixtos como trauma-cirugía y cuidados intensivos, trauma-anestesia y cuidados intensivos, cuidados intensivos coronarios, neumología-medicina crítica.

Ambos soluciones han ganado terreno y demostrado su eficacia en otros países, la primera particularmente en Europa y la segunda en Estados Unidos, donde se dispone de los recursos económicos y técnicos suficientes para que cada especialidad realice sus propios procedimientos.

En México, la mayoría de las escuelas de medicina crítica, incluidas las de los autores, se han inclinado por la primera solución, ${ }^{3-7}$ debido a las dificultades económicas para financiar el número de plazas requeridas para los cursos mixtos de posgrado y a las dificultades para concertar acuerdos con los 47 consejos de especialidades médicas agrupados en el Comité Normativo Nacional de Consejos de Especialidades Médicas (Conacem); es de notar que en Estados Unidos solo existen 24 consejos (boards).

Adquirir y compartir destrezas técnicas exclusivas de otras especialidades puede ser una alternativa para subsanar y complementar a mediano plazo el número de plazas de médicos especialistas sin incrementar el número de consejos de especialidad, a menos que esto sea estrictamente necesario. Este abordaje ha sido tan exitoso que actualmente muchos programas troncales de especialización como medicina de urgencias, medicina interna, cirugía general, anestesiología y ahora también ginecología y obstetricia, cardiología, neurología, neumología y otras efectúan rotaciones clínicas o adiestramientos en las unidades de cuidados intensivos.
Correspondencia:

*Jorge A. Castañón-González

E-mail: jorge.castanong @ gmail.com

0016-3813/0 2019 Academia Nacional de Medicina de Mexich

CC BY-NC-ND (http://creativecommons.org/licenses/by-nc-nd/4.0/).
Fecha de recepción: 23-10-2019

Fecha de aceptación: 11-12-2019

DOI: 10.24875/GMM.19005591
Gac Med Mex. 2020;156:1-3

Disponible en PubMed

www.gacetamedicademexico.com 


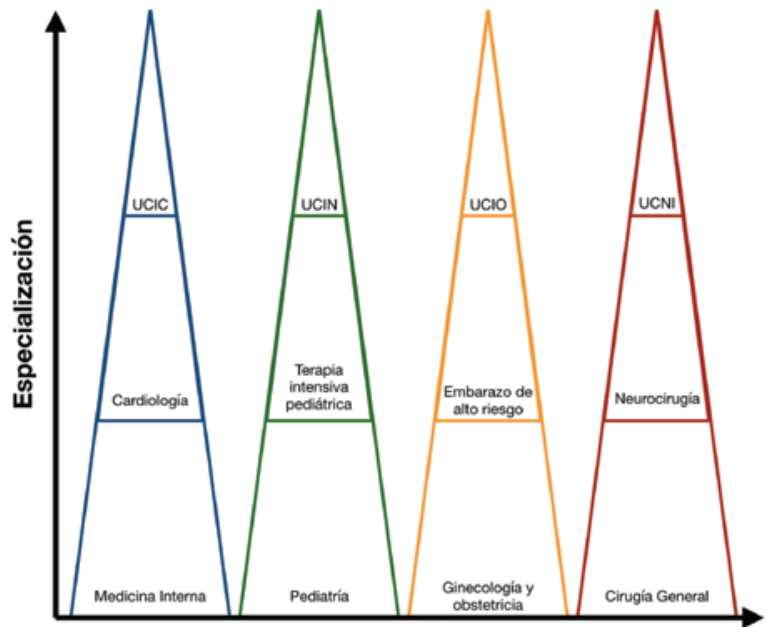

Campo de acción

Figura 1. Disposición vertical (tradicional) de las especialidades y subespecialidades en medicina. UCIC = Unidad de Cuidados Intensivos Cardiovasculares, UCIN = Unidad de Cuidados Intensivos Neonatales, UCIO = Unidad de Cuidados Intensivos Obstétricos, $\mathrm{UCNI}=$ Unidad de Cuidados Neurointensivos.

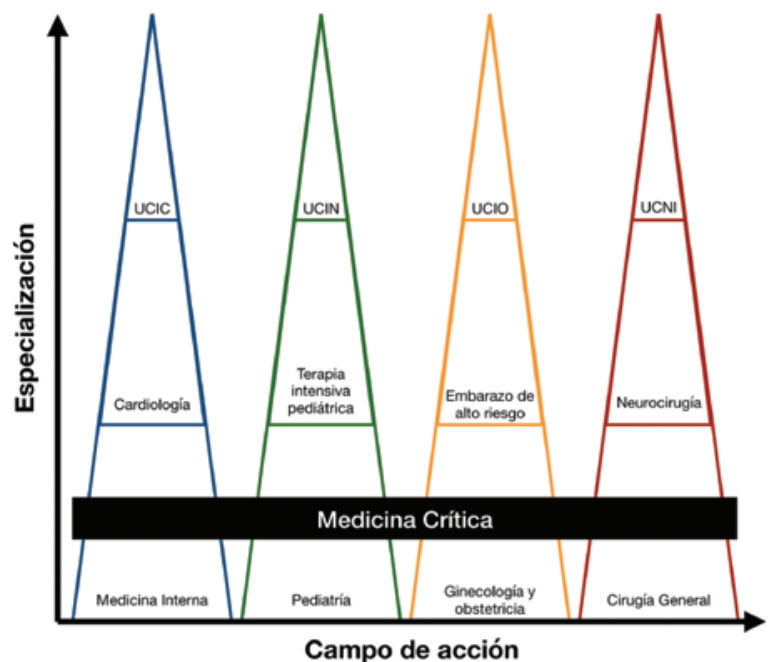

Figura 2. Disposición horizontal de la especialidad de medicina crítica en un hospital general. UCIC = Unidad de Cuidados Intensivos Cardiovasculares, UCIN = Unidad de Cuidados Intensivos Neonatales, $\mathrm{UCIO}=$ Unidad de Cuidados Intensivos Obstétricos, UCNI = Unidad de Cuidados Neurointensivos.

Debido a que la unidad de cuidados intensivos es el lugar en el hospital moderno donde se lleva a cabo el mayor número de procedimientos quirúrgicos después del quirófano, los beneficios de estos abordajes en cuanto adquisición de destrezas no se han hecho esperar, ya que la medicina crítica ha llevado a la cama del enfermo modalidades de diagnóstico como la ecografía, que ha agregado un "quinto" elemento a la exploración física clásica: la insonación se suma a la inspección, palpación, percusión y auscultación. ${ }^{8,9}$ Asimismo, hoy se considera indispensable la

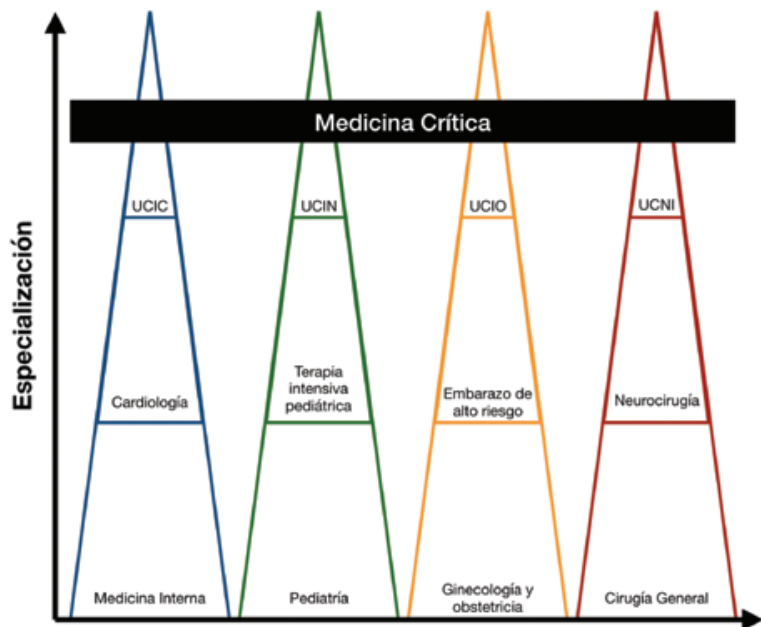

Campo de acción

Figura 3. Disposición horizontal de la especialidad de medicina crítica en un hospital de especialidades. UCIC = Unidad de Cuidados Intensivos Cardiovasculares, UCIN = Unidad de Cuidados Intensivos Neonatales, UCIO = Unidad de Cuidados Intensivos Obstétricos, $U \mathrm{CNI}=$ Unidad de Cuidados Neurointensivos.

broncoscopia para efectuar intubaciones traqueales difíciles, aspiración de secreciones, resolución de atelectasias y toma de muestras de lavado bronquioalveolar para el rastreo microbiológico en infecciones respiratorias bajas.

La evolución de la medicina crítica ha permitido la atención integral del paciente grave y en estado crítico en forma expedita y continua las 24 horas del día y los siete días de la semana; asimismo, ha coadyuvado a establecer diagnósticos, jerarquizar y privilegiar las interconsultas pertinentes y el tratamiento en forma temprana, eficiente y a menor costo al disminuir la estancia en la unidad de cuidados intensivos.

México es un país en desarrollo donde la tecnología de vanguardia no tiene una distribución homogénea en el territorio nacional, lo que condiciona que no esté al alcance de todos. Además, es un lugar donde el costo del día-cama en la unidad de cuidados intensivos es exorbitante debido a la dependencia del país en la tecnológica del extranjero: en 2018 se estableció en $\$ 35400$, el concepto unitario más caro del tabulador después de la cirugía. ${ }^{10}$ Afortunadamente, también es una nación donde las presiones que ejerce la sociedad y sus organismos oficiales frenan el costo cada vez mayor de la atención médica, regulado aún en gran parte por nuestra profesión, por lo que es responsabilidad de los especialistas en medicina crítica efectuar una reflexión profunda y detenida sobre el costo-beneficio de nuestra práctica para optimizar 
Ios recursos, limitados frente a una demanda de servicios que va en aumento en forma exponencial.

Es importante explicar a los médicos residentes que las observaciones clínicas basadas en una adecuada propedéutica, semiología y reconocimiento del contexto clínico son fundamentales y se complementarán con los estudios de gabinete, pero es el médico quien debe darles su valor clínico para la aplicación. La máxima de Guillermo de Ockham, filósofo escolástico del siglo XIII (Entia non sunt multiplicanda praeter necessitatem), menciona que los postulados no deben ser multiplicados sin necesidad o, en otras palabras, que es vano hacer con más recursos lo que se puede hacer con menos.

Es importante reiterar a los médicos residentes este principio de análisis lógico y de sentido común, ya que con frecuencia se pierde en el proceso educativo y es probable que se destruya deliberadamente en el proceso político. Creemos que, aún en condiciones laborales ideales, el especialista en medicina crítica debe ser austero por convicción en su práctica clínica y no por necesidad o moda institucional, y siempre basar sus decisiones en una sólida propedéutica clínica, un acervo de conocimientos amplio y con sustento en la evidencia científica más actualizada. ${ }^{11}$

\section{Bibliografía}

1. Ruelas-Barajas E, Alonso-Concheiro A, Alarcón-Fuentes A. Las especialidades. En: Consejo de Salubridad General, Secretaría de Salud, Academia Nacional de Medicina, editores. Futuros de la formación de recursos humanos para la salud en México 2005-2050. México: Ediciones Láser; 2008. pp. 53-58.

2. Castañón-González JA, Macías-Hernández AE, Polanco-González C, Domínguez-Cherit G. Broadening the scope of health care personnel in Critical Care Medicine. Cir Cir. 2011;79:451-452.

3. Ángeles-Valdés J, Bucay-Chouchleb M, Castañón-González JA, Fuentes-Pintado E. Ecocardiografía transesofágica en la unidad de cuidados intensivos. Rev Asoc Mex Med Crit Ter Intensiva. 1993;7(2):70-73.

4. Ángeles-Valdés J, Castañón-González JA, Bucay-Chouchleb M, Miranda-Ruíz R, Gómez-Velázquez AG. Utilidad de la ecocardiografía en la unidad de cuidados intensivos. Rev Asoc Mex Med Crit Ter Intensiva. 1993;7(5):172-177.

5. Deseáno-Estudillo JL, Castañón-González JA, Carbajal-Ramírez A, Castrejón-Román H, León-Gutiérrez MA. Espectro de velocidad del flujo sanguíneo cerebral por ultrasonido Doppler transcraneal en pacientes con criterios clínicos de muerte cerebral. Gac Med Mex. 2003;139:535-538.

6. Briones JC, Castañón JA, Díaz de León M, Briones CG. Velocidad de flujo sanguíneo cerebral en la preeclampsia-eclampsia. Prog Ostet Ginecol. (España) 2000;43:511-520.

7. Castañón-González JA, Castillo-Rodríguez G, Janet Tanus-Hajj J, Valencia-Jiménez E. Colecistitis aguda acalculosa en una unidad de cuidados intensivos multidisciplinaria. Gac Med Mex. 1996: 131:469-475.

8. Gómez-López L, Torres B, Bergé R, Aguirre O, Luis M, Sala-Blanch X. Medición ecográfica de parámetros anatómicos de la vía aérea superior en adultos. Rev Esp Anestesiol Reanim. 2018;65:495-503.

9. Pestana L. Insonar: cuestión de ángulos. Pánace@ 2001;2(3). Disponible en: www.medtrad.org/panacea/Indice. Consultado 2019 Feb 02.

10. Aprobación de los costos unitarios por nivel de atención médica actualizados al año 2018. Acuerdo ACDO.AS3.HCT.291117/275.P.DF del 29 de noviembre de 2018. Diario Oficial 2017 Dic 28.

11. Castañón-González JA. Reflexiones sobre la aplicación clínica de la tecnología en medicina crítica. Rev Asoc Mex Med Crit Ter Intensiva. 1994;8:40-41. 